\title{
Micro-scale Self-assembly of Long-range Ordered CuS Nanostructures
}

Shaobo Han ${ }^{1,2}$, Wei Liu ${ }^{1,2}$ and Kai Sun ${ }^{2}$

1. School of Physical Electronics, University of Electronic Science and Technology of China, Chengdu, China.

2. Department of Materials Science and Engineering, University of Michigan, Ann Arbor, MI, USA.

Copper sulfide $(\mathrm{CuS})$ due to its unique optical, electrical, and other physical and chemical properties, has been extensively studied in the areas of lithium batteries ${ }^{[1]}$ and solar cell ${ }^{[2]}$, sensing, photothermal therapy, imaging, supercapacitance, drug delivery, cathode materials, nonlinear optical materials, and catalysis.

Currently, varies of 3-D CuS have been successfully synthesized by hydrothermal and solvothermal methods. In these studies, the controlled synthesis of $\mathrm{CuS}$ caved superstructures are very interesting due to its highly geometrical shape ${ }^{[3,4]}$ However, long-range ordered structure of $\mathrm{CuS}$ has not been reported. In this study, a very simple, template-assistant solvothermal method was developed to synthesize microscale long-term orderly arrangement structure of $\mathrm{CuS}$. This structure is self-assembled by a large number of hexagonal $\mathrm{CuS}$ nanoplates.

The synthesis of $\mathrm{CuS}$ was performed according to the following procedures: $0.4 \mathrm{mmol} \mathrm{CuCl}_{2}$ was firstly dissolved in $50 \mathrm{~mL}$ deionized water with vigorous agitation to form a light green solution. Then, $1.2 \mathrm{~g}$ Polyvinylpyrrolidone (PVP) was added to the solution under magnetic stirring. 0.4 mmol thiourea was then added after all the substance was dissolved completely. The mixture was transferred into a $100 \mathrm{~mL}$ Teflon- lined stainless steel autoclave and heated at $100{ }^{\circ} \mathrm{C}$ for $15 \mathrm{~h}$. After cooling to room temperature naturally, the product was collected by centrifugation, washed with deionized water and ethanol, and then dried in a vacuum oven at $60{ }^{\circ} \mathrm{C}$ for $10 \mathrm{~h}$.

The structure and morphologies of the as-prepared $\mathrm{CuS}$ were recorded on a Hitachi S-4800 scanning electron microscope (SEM). XRD (X-ray powder diffraction) pattern was operated on a Japan RigakuD/Maxr-A X-ray diffractometer equipped with graphite monochromatized high-intensity $\mathrm{Cu} \mathrm{K \alpha}$ radiation $(\lambda=1.54178 \AA)$.

Fig. 1a displays typical SEM images of the as prepared hierarchical structure of CuS. X-ray diffraction was employed to understand the identity and phase purity of as-resulting products. As depicted in Fig. $1 \mathrm{~b}$, the entire diffraction pattern matches well with the standard data of covellite-type CuS with hexagonal lattice parameters of $\mathrm{a}=3.7920 \AA, \mathrm{b}=3.7920 \AA$ and $\mathrm{c}=16.3440 \AA$ (covellite, syn, JCPDS no. 06-0464).

Further detailed investigations of crystallographic features of as prepared CuS sample were performed by SAED and DF-TEM, as shown in Fig. 2. It indicates that the CuS hierarchical structure composed by two different crystallographic oriented CuS nanocrystals. [5]

References:

[1] Wang, X. X. et al. Journal of Power Sources 281 (2015), p. 185-191.

[2] Zhao, K. et al. Journal of Physical Chemistry C 118 (2014), p. 5683-5690.

[3] Wu, C.; Yu, S.-H.; Antonietti, M. Chemistry of Materials 18 (2006), p.3599-3601. 
[4] He, W. et al. Nanoscale 4 (2012), p. 3501-3506.

[5] A JEOL JEM-2010F was used in the study that was supported by the University of Michigan College of Engineering and NSF grant \#DMR-9871177.
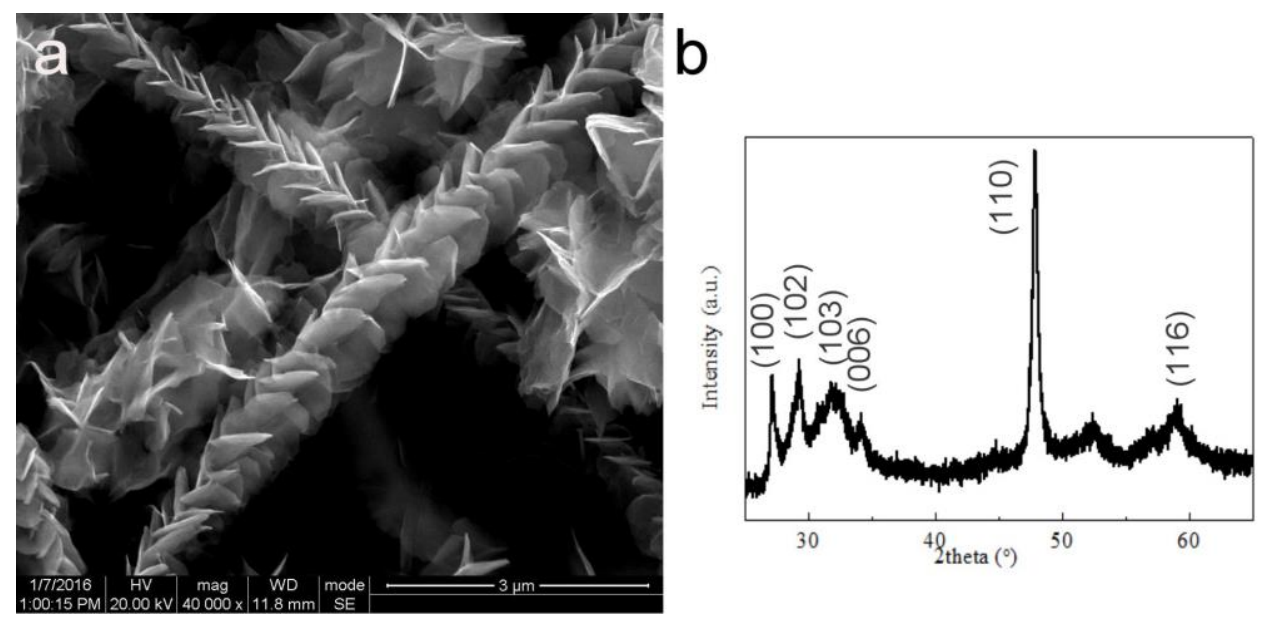

Figure 1. a) SEM image of the CuS sample, b) XRD pattern of the CuS sample, corresponding to JCPDS 06-0464.
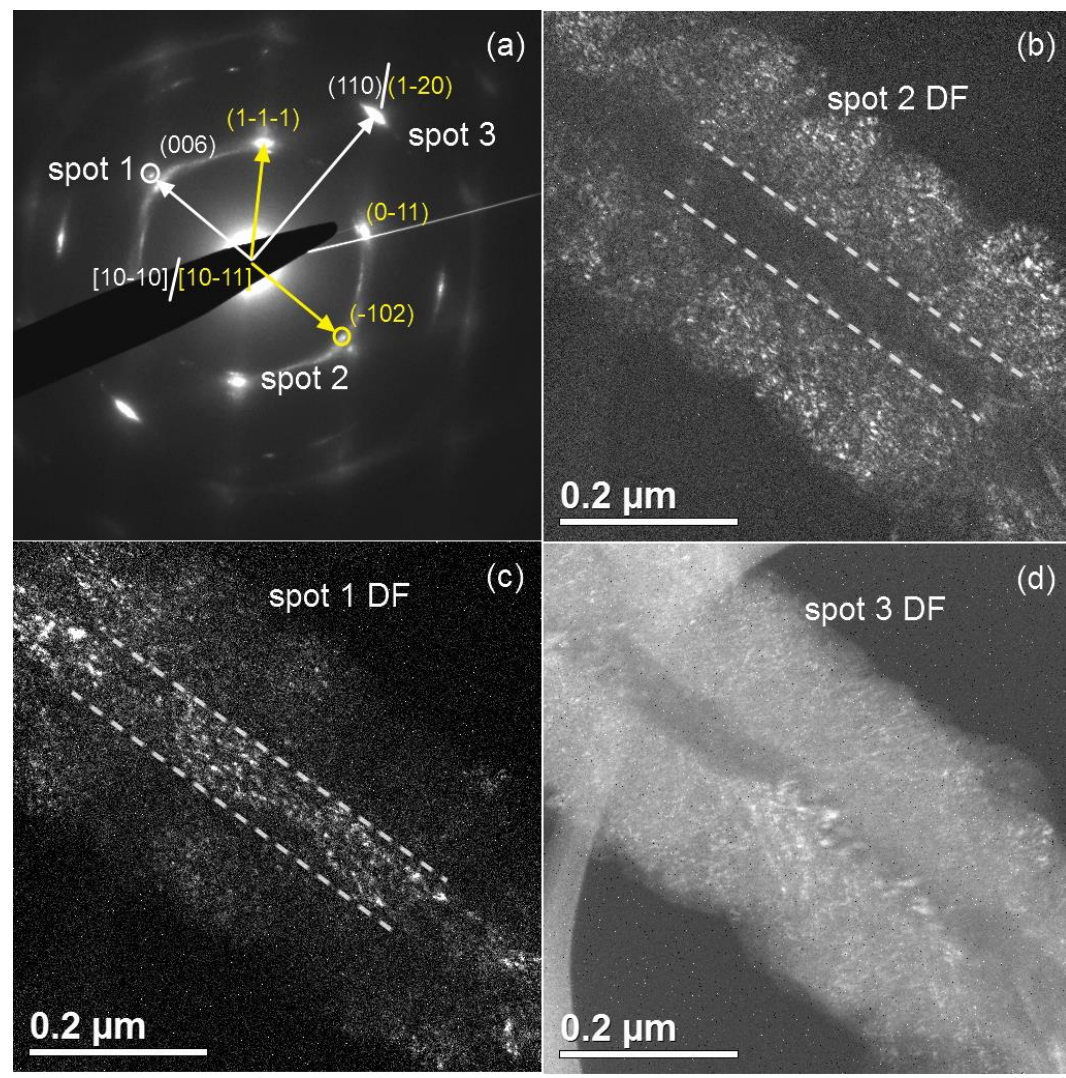

\section{$0.2 \mathrm{um}$}
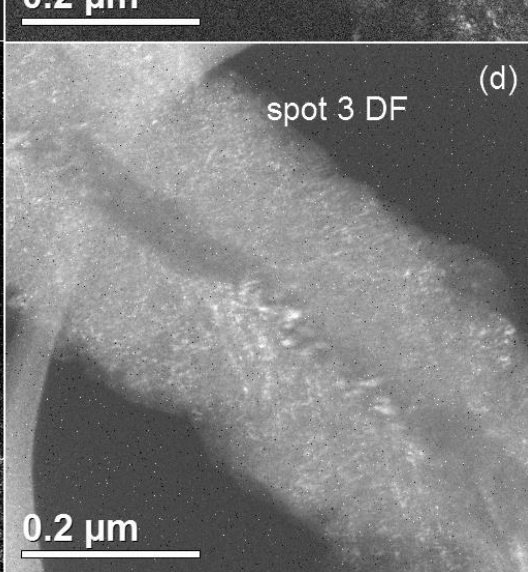

Figure 2. (a) SAED pattern of a single hierarchical structure CuS; (b), (c) and (d) are dark-field TEM images, corresponding to spot 2 , spot 1 and spot 3 respectively. 